\title{
Constraining the Dark Matter decay lifetime with very deep observations of the Perseus cluster with the MAGIC telescopes
}

\author{
J. Palacio* \\ Institut de Física d'Altes Energies (E-08193 Barcelona, Spain) \\ E-mail: jpalacio@ifae.es
}

M. Doro ${ }^{a, b}$, M. Vazquez Acosta ${ }^{c, d}$, P. Colin ${ }^{a}$, C. Maggio ${ }^{b, e}$, J. Rico ${ }^{f}$, F. Zandanel ${ }^{g}$ on behalf of the MAGIC Collaboration,

${ }^{a}$ Max Planck Institute for Physics (Munich, Germany),

${ }^{b}$ INFN-Padova (Padova, Italy),

${ }^{c}$ Instituto de Astrofísica de Canarias (E-38205 La Laguna, Tenerife, Spain),

${ }^{d}$ Universidad de La Laguna, Dpto. Astrofísica (E-38206 La Laguna, Tenerife, Spain),

${ }^{e}$ University of Padova (Padova, Italy),

${ }^{f}$ Institut de Fisica d'Altes Energies, (E-08193 Barcelona, Spain),

${ }^{g}$ GRAPPA Institute, University of Amsterdam, (1098 XH Amsterdam, The Netherlands)

\begin{abstract}
We present preliminary results on Dark Matter searches from observations of the Perseus galaxy cluster with the MAGIC Telescopes. MAGIC is a system of two Imaging Atmospheric Cherenkov Telescopes located in the Canary island of La Palma, Spain. Galaxy clusters are the largest known gravitationally bound structures in the Universe, with masses of $\sim 10^{15} \mathrm{M}_{\odot}$. There is strong evidence that galaxy clusters are Dark Matter dominated objects, and therefore promising targets for Dark Matter searches, particularly for decay signals. MAGIC has taken almost 300 hours of data on the Perseus Cluster between 2009 and 2015, the deepest observational campaign on any galaxy cluster performed so far in the very high energy range of the electromagnetic spectrum. We analyze here a small sample of this data and search for signs of dark matter in the mass range between $100 \mathrm{GeV}$ and $20 \mathrm{TeV}$. We apply a likelihood analysis optimized for the spectral and morphological features expected in the dark matter decay signals. This is the first time that a dedicated Dark Matter optimization is applied in a MAGIC analysis, taking into account the inferred Dark Matter distribution of the source. The results with the full dataset analysis will be published soon by the MAGIC Collaboration.
\end{abstract}

The 34th International Cosmic Ray Conference,

30 July- 6 August, 2015

The Hague, The Netherlands

\footnotetext{
* Speaker.
} 


\section{Introduction}

Dark Matter (DM) is the most abundant component of matter in the Universe according to the current concordance cosmological model dubbed $\Lambda \mathrm{CDM}$, making up to $85 \%$ of the total matter content [1]. DM can be composed of one or more kind of particles, but as of now we have only precisely determined the expected mass content through gravitational interaction, at many different scales, from the galactic to the cosmological one. The real nature of DM is unknown, included the mass range where we expect DM to be. One of the main questions is whether DM is totally secluded in a dark sector or it has some interaction with Standard Model particles. The Super-Symmetrical extension of the Standard Model of Particle Physics, for instance, gives a possible candidate, which is within the reach of the current experiments. Within this framework, a natural DM candidate arises as the lightest neutralino. This can be protected by a symmetry, and therefore stable and self-annihilating, or alternatively, very long-lived possibly providing decay signatures [2].

In this analysis, we investigate the possible signatures from DM decays by looking at a region in the sky where a very large density of DM is expected: the core of clusters of galaxies [3]. In this work, we focus on the Perseus galaxy cluster, observed in Very High Energy gamma rays with the MAGIC telescope for about $300 \mathrm{~h}$ in the years 2009-2015. Perseus is a cool-core cluster, the brightest in X-rays, and it is expected to contain about $10^{14} \mathrm{M}_{\odot}$ in form of DM.

The DM density distribution is usually parametrized following the Navarro-Frenk-White (NFW, [4]) profile, which reads as:

$$
\rho(r)=\frac{\rho_{s}}{\frac{r}{r_{s}}\left(1+\frac{r}{r_{s}}\right)^{2}}
$$

where $\rho(r)$ is the DM density as a function of the distance $r$ from the DM barycentre, and $r_{s}$ and $\rho_{s}$ are the characteristic scale radius and density of the source. In the case of Perseus we have taken $\left(r_{s}, \rho_{s}\right)=\left(0.477 \mathrm{Mpc}, 7.25 \times 10^{14} \mathrm{M}_{\odot} \mathrm{Mpc}^{-3}\right)$ following Ref. [5]. From the DM profile, the gamma-ray photon yield for decaying DM is computed using Eq. 1.2:

$$
\Phi(E, \Delta \Omega)=\frac{1}{4 \pi} \frac{1}{m_{\chi} \tau} \frac{d N_{\gamma}}{d E} \cdot J(\Delta \Omega) \quad \text { where } \quad J(\Delta \Omega)=\int_{\Delta \Omega} d \omega \int_{\text {los }} \rho(r(s)) d s,
$$

where $m_{\chi}$ and $\tau$ are the DM particle mass and lifetime, $d N_{\gamma} / d E$ is the number of photons globally produced as a result of the DM decay (e.g. through pion decay after hadronization of the quarks in the decay products) and $J(\Delta \Omega)$ is the so-called astrophysical factor in the solid angle $\Delta \Omega$, which is computed from the DM density profile as the integral over the line of sight and the solid angle of the matter density of Eq. 1.1.

The result of decaying DM in which we are interested here is the prompt production of gamma rays. The gamma-ray yield depends largely on the primary DM nature, both in terms of flux and spectral features. Fig. 1 (taken from Ref. [6]) clearly shows the features of the gamma-ray spectra for different DM decay scenarios. We also follow Ref. [6] here, to briefly introduce the decaying DM phenomenology. There are three classes of decaying DM modes according to the main products of the decay: ( $i)$ mainly leptonic, (ii) hadron and lepton, and (iii) mainly hadronic. Decaying DM models are also found in SUSY scenarios, if a mild violation of the R-parity (RPV = R-Parity Violation) is allowed, such that the Lightest Supersymmetric Particle (LSP) is not fully protected by the symmetry and may decay to Standard Model particles. There are several possibilities to 


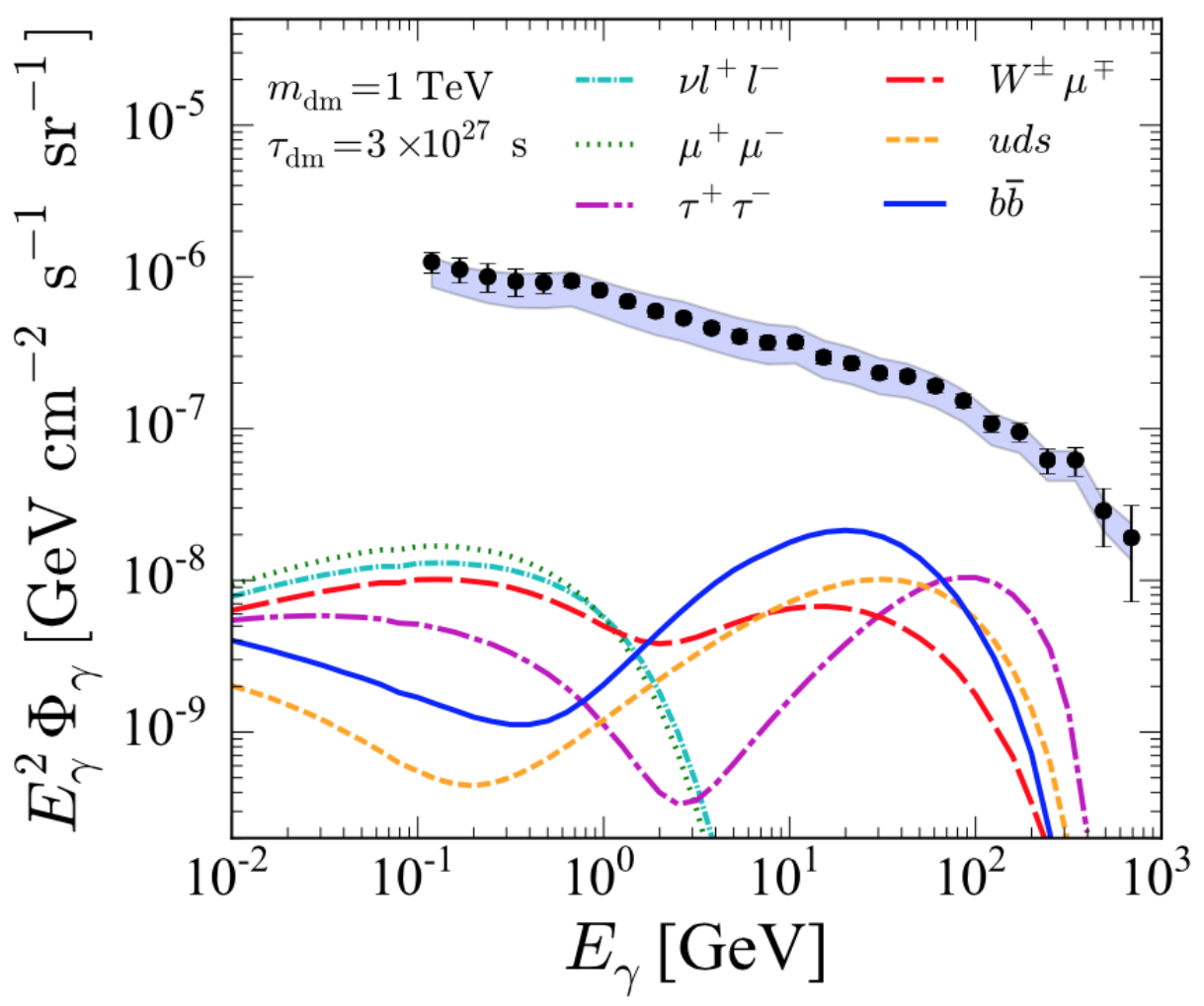

Figure 1: Gamma-ray spectra from different DM decay channel. To provide a photon yield the mass of DM is fixed at $1 \mathrm{TeV}$ and the decay lifetime to $3 \times 10^{27} \mathrm{~s}$. Several decay channels are shown: (a) $v e \mu^{-} \mu^{+}\left(v^{-} e \mu^{-} \mu^{+}\right)$and $v \mu e^{-} \mu^{+}\left(v^{-} \mu^{-} e \mu^{-}\right)$, (b) $\mu^{+} \mu^{-}$, (c) $\tau^{+} \tau^{-}$, (d) $W^{ \pm} \mu^{\mp}$, (e) $u d s$ ( $\bar{u} \bar{s} \bar{s}$, and (f) $b \bar{b}$. Data points with error bar and a band of the EGRB observed by Fermi-LAT is also shown. Figure is taken from Ref. [6].

actually do the RPV, and according to the details, the LSP can be a wino, a sneutrino, a gravitino or an axino. In all these cases, very energetic gamma rays would be generated during the decay process.

\section{The Perseus Cluster}

Perseus is a Galaxy Cluster located at $77.7 \mathrm{Mpc}(\mathrm{z}=0.0183)$ distance. With the above NFW parametrization, Eq. 1.1, in case of decaying DM, 90\% of the expected signal comes from a region of $1 \mathrm{deg}$, around the center of the cluster, which is 10 times larger than the telescope point spread function $\left(\sim 0.1^{\circ}\right)$. For this reason, the signal-search region, i.e. $\Delta \Omega$, must be extended and the exact aperture $\theta$ can be optimized considering that, under larger solid angles, the irreducible background from primary cosmic rays will also increase. We have therefore optimized the signal region through a quality-factor (Q-factor) defined as:

$$
Q(\Delta \Omega)=\frac{N_{\gamma}}{\sqrt{\left(N_{b k g}\right)}} \propto \frac{\int \operatorname{los}(\theta, \phi) \varepsilon_{\gamma}(\theta, \phi) \theta d \theta d \phi}{\sqrt{\int \varepsilon_{b k g}(\theta, \phi) \theta d \theta d \phi}},
$$


where $\operatorname{los}(\theta, \phi)=\int_{\text {los }} \rho(r(s)) d s$.

The numerator in Eq. 2.1 comes directly from $J(\Delta \Omega)$ in Eq. 1.2 convoluted with $\varepsilon_{\gamma}$, i.e. the efficiency of the camera for gamma-like events. The denominator is computed assuming a uniform flat background rate through all the camera, again taking into account the efficiency, $\varepsilon_{b k g}$, for background events. We assumed both efficiencies to be radially dependent, with respect to the center of the camera. As a result, the highest Q-factor is obtained for $\theta=0.35^{\circ}$. The total J-factor within this signal region is $J=5.6 \times 10^{18} \mathrm{GeV} \mathrm{cm}^{-2}$.

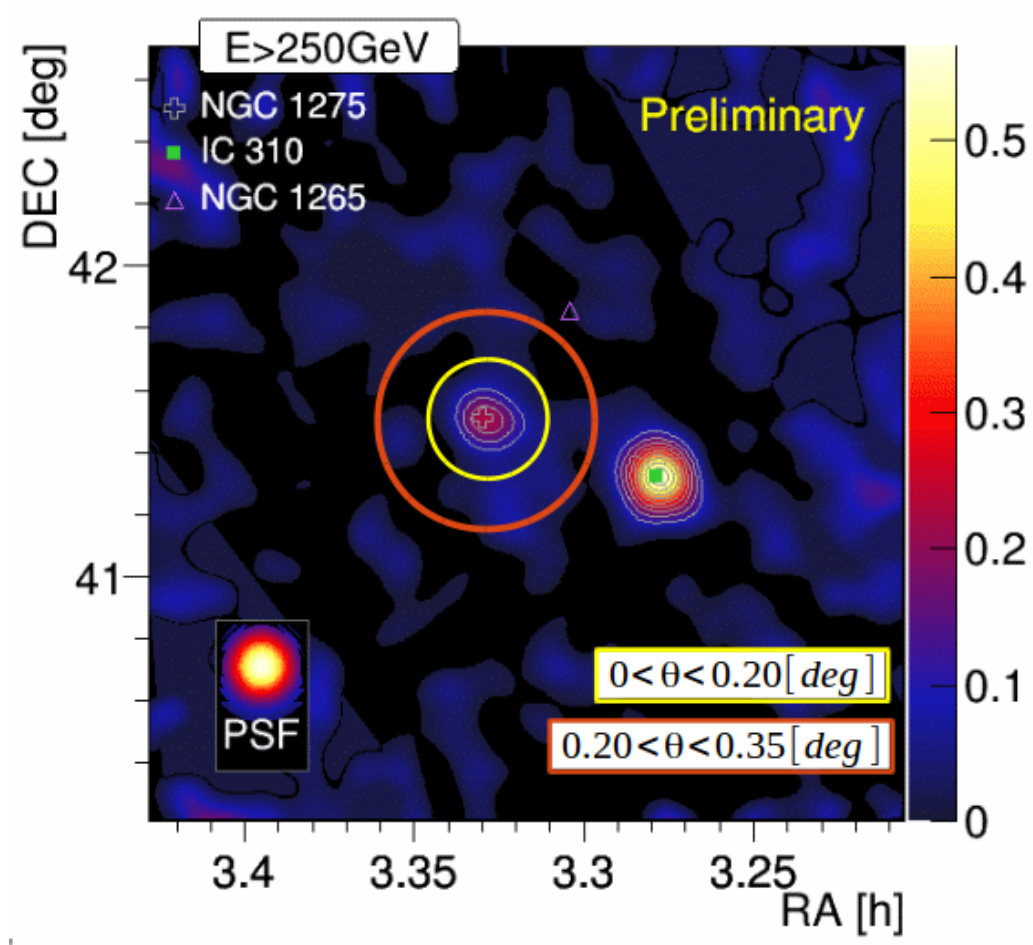

Figure 2: MAGIC sky map produced for the Perseus Cluster field of view. Some significance can be seen for two member galaxies of the cluster, NGC1275 and IC310. On top of that, we plot the two signal regions defined for the analysis (see text).

The tracking mode used in these observations, called wobble mode [18], allows us to obtain the background control region simultaneously with the signal region. For each pointing direction, signal and background are defined so that the regions where they are estimated have the same size and are at the same distance with respect to the center of the camera. In our case, the background events are obtained from a circular region of $0.35^{\circ}$ extension centered at a distance of $0.8^{\circ}$ away from the signal region. However, because of the large extension of the DM expected signal, there is a "leakage" of the DM expected signal in the background control region, albeit small, which we quantified as a few percent of the total expected signal and that is taken into account in our analysis. 


\section{MAGIC observation and data reconstruction}

MAGIC $^{1}$ is a system of two $17 \mathrm{~m}$ diameter tessellated dishes working in stereoscopic mode to observe the Cherenkov light coming from electromagnetic atmospheric showers initiated by VHE gamma rays impinging the Earth. MAGIC is currently the instrument reaching the lowest energy threshold among ground based gamma rays detectors [7, 8]. It is located in the Canary island of La Palma (Spain) and is taking data since 2004. Its core science is focused on the study of the cosmic ray origin in either galactic or extragalactic targets, but it is well-known that cosmic gamma rays constitute also a probe for several fundamental physics quests, including dark matter (see, e.g. [9]). The Perseus campaigns proved very fruitful, providing the strongest limits on CR acceleration in the core of the cluster and the cosmic ray to thermal pressure [10,11, 12], the AGN NGC1275 at the center of the cluster was clearly detected and modeled $[13,14]$, as well as interesting results came from the peculiar radio-galaxy IC310 located at $0.6 \mathrm{deg}$ from the Perseus center, bringing an interesting clue in the mechanism of acceleration of cosmic rays close to black holes $[15,16,17]$. Here, we focus on the signatures from DM decays coming from the cluster.

During the 6-year observational campaigns, MAGIC has gone through several hardware upgrades, and therefore different data samples are analyzed using different instrumental response function (IRFs, mainly effective area and energy migration tables), the details of which will be reported in the incoming publication of the MAGIC collaboration, under preparation. For each data sample, pixel-wise data are cleaned and combined into global event parameters expressed with the Hillas formalism [19], and assigned a parameter called "hadronness" built through a classification method called Random Forest (RF), through comparison with a tuned Monte Carlo sample. The RF also assigns the events estimated energy and direction values. In the further analysis steps, data is analyzed through the full likelihood method described in [20]. The input of the (binned) likelihood are: the list of the energy of photons coming from the signal region and the background control region (after various quality cuts, specially the hadronness one), as well as the IRFs above mentioned for that period. The likelihood is built defining the null hypothesis as the case where no excess over the background is found, while the test hypothesis considers the flux computed using Eq. 1.2 and under the hypotheses of two different pure DM decays: one into $b \bar{b}$ and one into $\tau^{+} \tau^{-}$ often used in the literature as representative of soft and hard spectra respectively. Other channels have a spectral distribution normally in between these two. The analytical formulas for the photon yield in the two cases are taken from [22].

To build the correct IRFs for each subsample, we developed a new tool to selectively choose from a diffuse sample of Monte Carlo (globally covering the 3 deg of the camera FoV) events, those that match the expected distribution of signal events in the data. This procedure will also be described in the incoming MAGIC publication.

Summarizing, we have prepared $7($ Period $) \times 2(\mathrm{Zd}) \times 2($ Ring $)=28$ different subsamples, each of them with different IRFs, which are later on combined together using the Full Likelihood formalism. In the above list, "Period" are the stable periods of the Telescopes, the two bins in "zenith angle (Zd)" are appropriate because both the effective area and energy resolution are affected and "Ring" stands for the division of our signal region into two concentring rings. The reason for this last spatial binning is first, the dependence of the camera response at large distances with respect

\footnotetext{
${ }^{1}$ http: //magic.mpp.mpg.de
} 
to its center, and most important, NGC1275's expected emission, lying at the center of the cluster. The size of the first bin was chosen so that more than $95 \%$ of NGC1275's emission is expected to be in the inner ring [8]. This astrophysical contamination needs a special treatment in the likelihood analysis and details on this will be reported in the upcoming full data analysis publication.

\section{Results}

We present the results on the decay life time constraints obtained with a small subsample of the Perseus Cluster data taken with MAGIC. We analyze data taken during the period from 2013.07.27 to 2014.08.05, with zenith angles between $5^{\circ}$ to $50^{\circ}$ and only one pointing mode (we have two pointing modes). This sums up to 1 (Period) $\times 2(\mathrm{Zd}) \times 1$ (Ring $)=2$ subsamples with different IRFs. We have made all the consistency checks for the robustness of the reconstruction, which will be later on applied to the complete data set analysis.

We found no excess in this region of the sky. The total J-factor in the case of decaying DM corresponds to about $J(0.2<\theta<0.35)=1.8 \times 10^{18} \mathrm{GeV} \mathrm{cm}^{-2}$. By fixing a DM mass and a decay channel, we derive, from our null detection, a lower limit on the DM decay lifetime using eq. 1.2. For the decay channel, we used the analytical computation of [22] (based on PITHYA), for two pure decay channels: $b \bar{b}, \tau^{+} \tau^{-}$. We repeat the procedure for several DM masses in the range 160 $\mathrm{GeV}$ to $200 \mathrm{TeV}$ and compute again the lower limits.

Our results, obtained with a subset of $12.40 \mathrm{~h}$, provide a lower limit on the DM decay lifetime of $10^{24} \mathrm{sec}$ at $100 \mathrm{GeV}$ and $10^{26} \mathrm{sec}$ at $1 \mathrm{TeV}$. With the full data sample analysis we expect an improvement in sensitivity of a factor 4 with compared to the present results.

\section{Summary and Conclusions}

We report here the lower limits on the dark matter decay lifetime obtained with a subset of data taken with the MAGIC telescope of the Perseus galaxy cluster. The full dataset comprises $300 \mathrm{~h}$ in 6 years with MAGIC and will be published soon. A binned likelihood method will be used to combine results taken under different experimental conditions, including different data taken periods, different observation modes, different zenith angles and radial distances from the center of the source.

\section{Acknowledgement}

We would like to thank the Instituto de Astrofísica de Canarias for the excellent working conditions at the Observatorio del Roque de los Muchachos in La Palma. The financial support of the German BMBF and MPG, the Italian INFN and INAF, the Swiss National Fund SNF, the ERDF under the Spanish MINECO (FPA2012-39502), and the Japanese JSPS and MEXT is gratefully acknowledged. This work was also supported by the Centro de Excelencia Severo Ochoa SEV-2012-0234, CPAN CSD2007-00042, and MultiDark CSD2009-00064 projects of the Spanish Consolider-Ingenio 2010 programme, by grant 268740 of the Academy of Finland, by the Croatian Science Foundation (HrZZ) Project 09/176 and the University of Rijeka Project 13.12.1.3.02, by the DFG Collaborative Research Centers SFB823/C4 and SFB876/C3, and by the Polish MNiSzW grant 745/N-HESS-MAGIC/2010/0.

\section{References}

[1] G. Bertone, D. Hooper and J. Silk, "Particle dark matter: Evidence, candidates and constraints," Phys. Rept. 405 (2005) 279 [hep-ph/0404175]. 
[2] J. L. Feng, "Dark Matter Candidates from Particle Physics and Methods of Detection," Ann. Rev. Astron. Astrophys. 48 (2010) 495 [arXiv:1003.0904 [astro-ph.CO]].

[3] Voit, G. Mark "Tracing cosmic evolution with clusters of galaxies" Rev.Mod.Phys. 77 (2005) 207-258 [arXiv:0410173 [astro-ph]]

[4] J. F. Navarro, C. S. Frenk and S. D. M. White, Astrophys. J. 462 (1996) 563 [astro-ph/9508025].

[5] M. A. Sanchez-Conde, M. Cannoni, F. Zandanel, M. E. Gomez and F. Prada, JCAP 1112 (2011) 011 [arXiv:1104.3530 [astro-ph.HE]].

[6] S. Ando and K. Ishiwata, "Constraints on decaying dark matter from the extragalactic gamma-ray background,” JCAP 1505 (2015) 05, 024 [arXiv:1502.02007 [astro-ph.CO]].

[7] J. Aleksić, S. Ansoldi, L. A. Antonelli, P. Antoranz, A. Babic, P. Bangale, M. Barcelo and J. A. Barrio et al., "The major upgrade of the MAGIC telescopes, Part I: The hardware improvements and the commissioning of the system," arXiv:1409.6073 [astro-ph.IM].

[8] J. Aleksić et al. [MAGIC Collaboration], “The major upgrade of the MAGIC telescopes, Part II: The achieved physics performance using the Crab Nebula observations," arXiv:1409.5594 [astro-ph.IM].

[9] M. Doro et al. [CTA Collaboration], Astropart. Phys. 43 (2013) 189 [arXiv:1208.5356 [astro-ph.IM]].

[10] J. Aleksić et al. [MAGIC Collaboration], "MAGIC Gamma-Ray Telescope Observation of the Perseus Cluster of Galaxies: Implications for Cosmic Rays, Dark Matter and NGC 1275," Astrophys. J. 710 (2010) 634 [arXiv:0909.3267 [astro-ph.HE]].

[11] J. Aleksić et al. [MAGIC Collaboration], "Constraining Cosmic Rays and Magnetic Fields in the Perseus Galaxy Cluster with TeV observations by the MAGIC telescopes," Astron. Astrophys. 541 (2012) A99 [arXiv:1111.5544 [astro-ph.HE]].

[12] P. Colin et al. [MAGIC Collaboration], "Detection of very high energy gamma-ray emission from NGC 1275 by the MAGIC telescopes," These procs. ID xx

[13] J. Aleksić et al. [MAGIC Collaboration], "Detection of very high energy gamma-ray emission from NGC 1275 by the MAGIC telescopes," Astron. Astrophys. 539 (2012) L2 [arXiv:1112.3917 [astro-ph.HE]].

[14] J. Aleksić et al. [MAGIC Collaboration], "Contemporaneous observations of the radio galaxy NGC 1275 from radio to very high energy $\gamma$-rays,” Astron. Astrophys. 564 (2014) A5 [arXiv:1310.8500 [astro-ph.HE]].

[15] J. Aleksić et al. [MAGIC Collaboration], "Detection of very high energy gamma-ray emission from the Perseus cluster head-tail galaxy IC 310 by the MAGIC telescopes,” Astrophys. J. 723 (2010) L207 [arXiv:1009.2155 [astro-ph.HE]].

[16] J. Aleksić et al. [MAGIC Collaboration], "Rapid and multiband variability of the TeV bright active nucleus of the galaxy IC 310,” Astron. Astrophys. 563 (2014) A91 [arXiv:1305.5147 [astro-ph.HE]].

[17] J. Aleksić et al. [ Collaboration], "Black hole lightning due to particle acceleration at subhorizon scales,” Science 346 (2014) 1080 [arXiv:1412.4936 [astro-ph.HE]].

[18] V. P. Fomin, A. A. Stepanian, R. C. Lamb, D. A. Lewis, M. Punch and T. C. Weekes, "New methods of atmospheric Cherenkov imaging for gamma-ray astronomy. 1: The False source method," Astropart. Phys. 2 (1994) 137.

[19] A. M. Hillas, "Cerenkov light images of EAS produced by primary gamma", Proceedings of the 19th International Cosmic Ray Conference. Vol.3, La Jolla U.S.A. (1985), pg. 445. 
[20] J. Aleksić, J. Rico and M. Martinez, "Optimized analysis method for indirect dark matter searches with Imaging Air Cherenkov Telescopes,” JCAP 1210 (2012) 032

[21] J. Aleksić et al. [ Collaboration], "Optimized dark matter searches in deep observations of Segue 1 with MAGIC" title = "Optimized dark matter searches in deep observations of Segue 1 with MAGIC", JCAP 1402 (2014) 008

[22] J. A. R. Cembranos, A. de la Cruz-Dombriz, A. Dobado, R. A. Lineros and A. L. Maroto, "Photon spectra from WIMP annihilation,” Phys. Rev. D 83 (2011) 083507 [arXiv:1009.4936 [hep-ph]].

[23] L. Dugger, T. E. Jeltema and S. Profumo, JCAP 1012 (2010) 015 [arXiv:1009.5988 [astro-ph.HE]]. 